\title{
Von der Schwierigkeit, ein Territorium zu konstituieren und zu behaupten Der Streit zwischen Comburg und Hohenlohe um die Vorherrschaft über das Dorf Mistlau
}

Teil 2: von 1788 bis 1802

von ULRICH FröHNER

\section{Ein merkwürdiger Brief}

Am 13. Mai 1788 erhielt Fürst Christian Friedrich Carl zu Hohenlohe-Kirchberg einen Brief von Johann Godefried Lothar Franz Freiherr von Greiffenclau zu Vollrads, den dieser in seinem eigenen Namen als Dekan (in den Quellen des 18. Jahrhunderts meist: Dechant $)^{1}$ und im Namen des Kapitels des Ritterstifts Comburg geschrieben hatte. ${ }^{2}$ Christian Friedrich Carl regierte Hohenlohe-Kirchberg seit 1764. Er war 178859 Jahre alt und ein gewissenhafter und fleißiger Regent. Mit seiner kleinen, gut lesbaren Handschrift schrieb er selbst Aktenvermerke, machte Randbemerkungen zu Stellungnahmen seiner Kanzlei und korrigierte deren Briefentwürfe. Zum Brief des Ritterstifts befahl er in einem Memorandum am folgenden Tag seiner Kanzlei, dem Dekan zu antworten. Es ging um Rechte in Mistlau, die seit langem zwischen Hohenlohe und Comburg strittig waren. ${ }^{3}$

Wegen Mistlau war Comburg in einen schon eineinhalb Jahrhunderte währenden Streit mit Hohenlohe-Kirchberg verwickelt darüber, wer dort die Oberherrschaft hatte. Zumeist beschränkte sich dieser Streit auf den Austausch von Protestbriefen, wenn Kirchberg einen „Frevler“" abgeurteilt hatte und Comburg meinte, seine Rechte seien dadurch verletzt worden. Der Fürst nun wunderte sich darüber,

1 Greiffenclau war von 1771 bis zum Ende des Ritterstifts 1802 Dechant. Im Jahr 1788 war er 50 Jahre alt. http://www.dr-bernhard-peter.de/Heraldik/aktuell/galerien3/galerie1903.htm

2 Alle im Folgenden zitierten Dokumente sind verwahrt im HZA unter Ki 10 17A Nr. 56. Sie sind in der Akte durchnummeriert. Es wird im Folgenden jeweils die Akten-Nummer (ohne Signatur) angegeben; der Brief des Dekans also als AN 1.

3 Vgl. dazu Ulrich Fröhner: Von der Schwierigkeit, ein Territorium zu konstituieren und zu behaupten. Der Streit zwischen Comburg und Hohenlohe um die Vorherrschaft über das Dorf Mistlau. Teil 1: Ein Prozess vor dem Reichskammergericht von 1688 bis 1700. In: WFr 100 (2016), S. 107130 . 


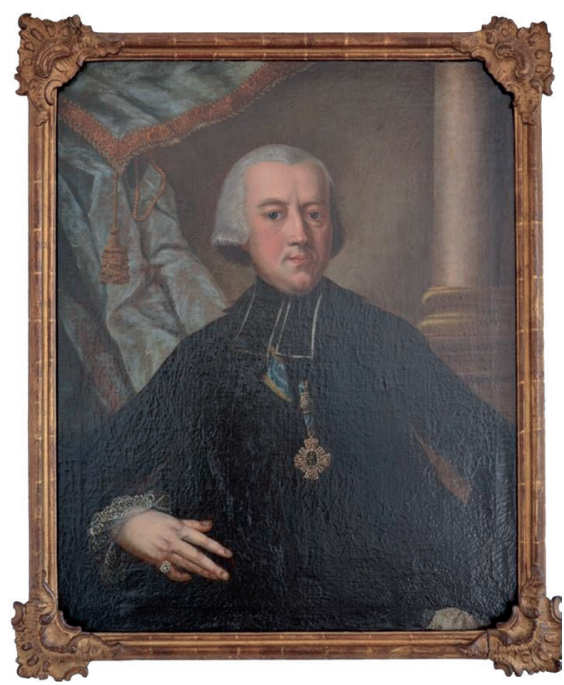

Dechant Johann Godefried Lothar

Franz Freiherr von Greiffenclau zu Vollrads

(Bild: Franz Zimmermann).

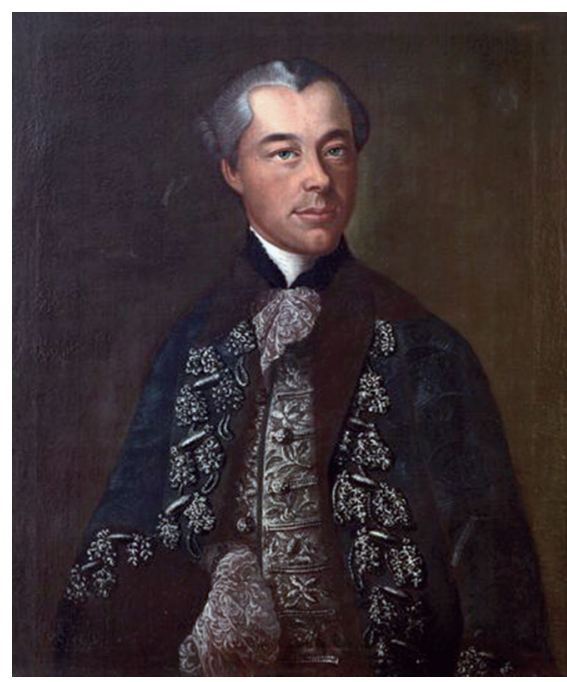

Fürst Christian Friedrich Carl zu Hohenlohe-Kirchberg (Bild: Historisches Museum Schloss Kirchberg).

dass Comburg hier auf so alte Geschichten zurückkam - und dies in seinem so höflichen Ton tat, dass man vermuten könne, es stecke ein verdecktes Friedensangebot dahinter. Er bat seine Kanzlei, dazu Stellung zu nehmen. ${ }^{4}$

\section{Zwei Stellungnahmen}

Dies geschah in zwei Stellungnahmen. Die erste stammt vom Geheimen Hofrat Cunradi. Er war Vorsteher der Regierung von Hohenlohe-Kirchberg. Diese bestand dazumal aus acht Personen: Aus Cunradi selbst, dem Assessor Johann Friedrich Wiedmann, einem Sekretär, einem Registrator, einem Accesisten (Sekretärs-Lehrling), einem Kanzlisten, einem Kanzleidiener und einem Kanzleiboten $^{5}$ - lässt man die Hilfskräfte beiseite, also aus zwei Personen, dem Hofrat und dem Assessor.

Cunradi war damals 64 Jahre alt und stammte aus der Familie Conrad/Cunradi, die den Hohenlohern in Kirchberg und Langenburg seit dem 17. Jahrhundert als Vögte, Amtmänner und Räte diente. Im Sandelschen Museum in Kirchberg be-

4 AN 2; vgl. Anhang 1 zur vorliegenden Arbeit.

5 Die untergeordneten Chargen nach dem Hofkalender von 1801, HZA GA 93, Bd 16 4-7; die Kanzleibesetzung dürfte sich 1788 kaum von der im Jahr 1801 unterschieden haben. 
findet sich ein Porträt von ihm, das einen alten, gütigen, aber durchaus verschmitzten Mann zeigt.

Cunradi erstellte am gleichen Tag ein Gutachten für den Fürsten. ${ }^{6}$ Er verwies darauf, dass man das comburgische Schreiben nicht erwartet habe, da man gegenwärtig wegen dieses Dorfs nicht in Strittigkeit mit dem Ritterstift verwikelt sei. Vielmehr beträfen die angegebene $\mathrm{Be}$ schwerden ältere Vorfälle, wegen derer die hohenlohische Regierung sich gegen Comburg schon hinlänglich gerechtfertigt habe. Wegen des versöhnlichen Tons des comburgischen Schreibens und weil diesmal nicht, nach

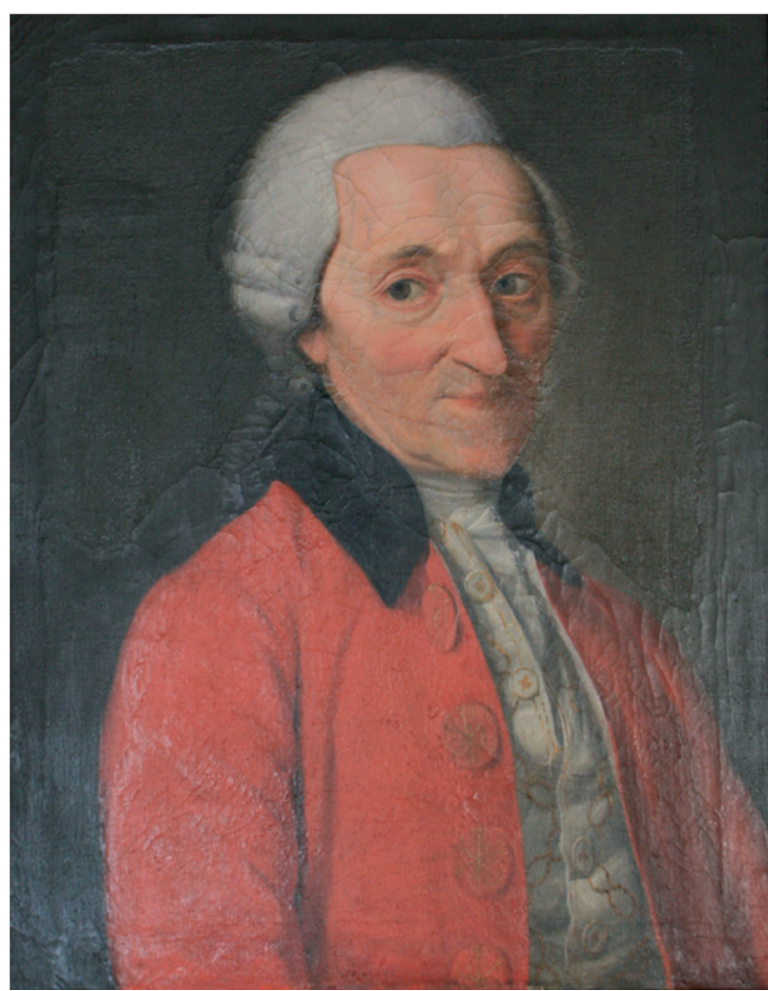

Hofrat Johann Christoph Cunradi (Bild: Ulrich Fröhner). sonstiger Gewonheit, am Ende mit Proceß am Reichsgericht gedrohet werde, vermute man, dass Comburg einen Ausgleich vorhabe und wohl die Mistlauer Strittigkeiten mit ähnlichen Differenzen im Dorf Gaggstatt ausgleichen wolle. Cunradi meinte auch, er habe zuverlässig gehört, dass sich schon bei der vorigen Kapitelversammlung einige der Teilnehmer kritisch zur neuen Prozesswut ${ }^{7}$ der comburgischen Kanzlei geäußert hätten. Er schlug deshalb ein Sondierungsgespräch zwischen Comburg und Kirchberg vor, um abzuklären, ob Comburg tatsächlich vergleichswillig sei. Vielleicht ließe sich dann ein Handel in der Weise eingehen, dass Kirchberg in Mistlau, wo seine Verhandlungsposition stärker sei, etwas nachgebe und dafür Frieden und eine Einstellung des Prozesses wegen Gaggstatt erhalte. Würde das Sondierungsgespräch ein positives Ergebnis brin-

6 AN 3.

7 Comburg hat zu dieser Zeit drei Prozesse beim Reichskammergericht laufen: den mit Hohenlohe-Kirchberg wegen der Territorial-Herrschaft in Gaggstatt und zwei Prozesse mit Schwäbisch Hall wegen ähnlicher Fragen. Alexander Brunotte / Raimund J. Weber: Akten des Reichskammergerichts im Hauptstaatsarchiv Stuttgart. Stuttgart 2005, Nr. 627, Nr. 616 und 617. 
gen, dürften dann die übrigen Ganerben - nämlich Langenburg und Ingelfingen - in Gestalt von Geheimrat von Zwanziger zuzuziehen sein.

Wesentlich kritischer als die Cunradis fiel die Stellungnahme des Assessors Wiedmann aus. ${ }^{8}$ Er unterstellte Comburg, das er höchst negativ beurteilte, nur taktische Raffinesse. Wiedmann vermutete, dass auch in der Mistlauer Sache ein Prozess in Vorbereitung sei und der Brief Comburgs nur beweisen sollte, dass mit Kirchberg eine friedliche Einigung nicht möglich sei. Er habe im Übrigen in den Akten überhaupt keinen Vorfall im Jahr 1766 finden können, auf den sich Comburg beziehen könne. Wiedmann legte seinem Schreiben noch das Urteil des Reichskammergerichts von 1566 bei, in welchem Kirchberg die vogteiliche Obrigkeit über Mistlau zugesprochen worden war und schloss mit der Empfehlung, sich im Antwortschreiben versöhnlich zu geben, Comburg aufzufordern, eine Aufstellung seiner Beschwerdepunkte gegen Kirchberg einzureichen und dann in einer gemeinsamen Besprechung Wege zu suchen, sie zu beseitigen. Dann werde man sehen, wie Comburg reagiere - und habe für einen eventuellen Prozess nichts verloren.

\section{Vorsichtige Friedensfühler}

Nach dieser Devise - aber mit gebotener Vorsicht - wurde dann auch weiter verfahren. Am 18. Mai bedankte sich der Fürst einerseits für die comburgischen Komplimente, verwahrte sich aber andrerseits gegen die Comburger Vorwürfe. Er verwies auf das Urteil von 1566, das er vorher nicht gekannt und nun mit Freude zur Kenntnis genommen habe, und schloss mit dem Hinweis, Comburg möge doch die vermeintlichen Beschwerden zusammentragen lassen. Dann würde er sie untersuchen lassen und nach einem gütlichen Ausgleich streben.

Die am 30. Juni 1788 abgeschickte comburgische Antwort traf am 1. Juli in Kirchberg ein und fiel positiv aus. ${ }^{9}$ Comburg wies zwar den Vorwurf zurück, es gefährde den nachbarlichen Frieden, ging aber auf das fürstliche Angebot ein, einen gütlichen Ausgleich zu suchen. Einzelheiten sollte der Comburger Syndikus, der Licentiat Weis, mit Cunradi aushandeln.

Weis leitete die comburgische Kanzlei. Er war - im Gegensatz zu seinen Auftraggebern - ein Bürgerlicher und hatte Jura studiert. Offenbar war er an pragmatischen Lösungen interessiert, während seine adligen Auftraggeber oft das Grundsätzliche, die Ehre und das Rechtbehalten in den Vordergrund stellten. Weis war gleichzeitig Geheimer Rat beim Fürsten von Hohenlohe-Bartenstein. Der gehörte allerdings zum katholischen Zweig der Hohenloher - ein Hohenloher war er freilich auch.

8 AN 5.

9 AN 7. 


\section{Ein Gespräch wird vorbereitet}

Nun galt es, die Gespräche vorzubereiten. Kirchberg zog für die Seite der Hohenloher den Geheimen Hofrat Zeller von Langenburg hinzu. Er musste zuerst die Akten studieren. Diese zu finden war nicht ganz einfach, wie Cunradi seinem Fürsten am 9. Oktober 1788 mitteilte. Cunradi klagte, dass der Registrator sie nach langer Suche unter einem Wust von anderen unordentlich untereinander liegenden schriftlichen Sachen vorgefunden habe. ${ }^{10}$

Offenbar hatte man diese Streitigkeiten vorher in Kirchberg nicht gerade als wichtig angesehen. Worum ging es in diesen Akten?

1766 hatten drei junge Burschen aus Anlass einer Hochzeit auf der Straße mutwillig geschossen. Kirchberg wollte sie dafür zur Rechenschaft ziehen und der Kirchberger Amtsknecht betrat dazu Comburger Häuser. Da Kirchberg die Burschen offenbar nicht belangen konnte, bat es Comburg um Auslieferung. Gegen beides protestierte Comburg. Nicht lange danach, am 8. September 1766, ließ bei der Kirchweih in Mistlau dann der Kirchberger Rat und Amtmann Hochheimer einen der Übeltäter festnehmen und erst wieder laufen, nachdem er für sich und seine zwei Kumpane einen Reichstaler Strafe bezahlt hatte. Auch dagegen hatte Comburg protestiert.

Beim zweiten Vorfall war am 10. Juni 1786 ein Kirchberger Soldat in das Haus des Comburger Untertanen Reu eingetreten und hatte einen herumziehenden Spielmann, der sich dort aufgehalten hatte, des Dorfes verwiesen. Damit hatte er Comburger Rechte verletzt - was in diesem Fall Kirchberg auch ohne Weiteres zugegeben und beteuert hatte: Man habe zwar den Soldaten, welche zur Land-Patrouille beordert werden, die Anweisung [gegeben], die Bettel-Leuthe auf den Straßen, und von den Häußern abzutreiben, nicht aber in die Häuser selbst einzugehen, und sie dort aufzusuchen.

Comburg war mit dieser Antwort zufrieden gewesen und hatte sich sogar für die Durchführung der Streife bedankt, da Comburg selbst wegen der Entfernung und wegen Geldmangels nicht so oft selbst streifen könne. Comburg wollte aber nicht hinnehmen, dass der oben genannte Soldat - und neulich ein Amtsknecht - in Worte ausgebrochen, daß Comburg hier-zu Mistlau - nichts zu bestellen habe und verwahrte sich gegen solche Gassen-Worte.

Soweit zu den Vorfällen, die Anlass zu dem Comburger Brief gegeben hatten. Zurück ins Jahr 1788. Cunradi schickte die Akten an den Fürsten und schrieb dazu, von Weis habe man noch nichts gehört wegen eines Besprechungstermins - und der Fürst schrieb an den Rand, dann solle vorerst auf keinen Fall etwas von Kirchberger Seite unternommen werden. Wenn Kirchberg sich hier zu eifrig zeige, könne in Comburg der Verdacht aufkommen, Kirchberg fühle sich seiner Sache in Gaggstatt nicht sicher genug. 
Um was aber war es beim Prozess wegen Gaggstatt gegangen? Comburg ,und die Freiherren von Seckendorf und von Crailsheim als Ganerben zu Gaggstatt" hatten gegen Hohenlohe-Kirchberg geklagt ,wegen: Verletzung der zur Ganerbenschaft Gaggstatt gehörenden gemeinschaftlichen Rechte (Dorfherrschaft, Landeshoheit einschl. Episkopalrecht, Vogtei und Gerichtsbarkeit auf Gassen und Straßen sowie Hirtenstab) durch Bekl., der sich Stellung eines obersten Dorf- und Territorialherren sowie des alleinigen Inhabers des Hirtenstabs anmaßt, i.e. durch Bestrafung der Frevel auf der Gasse, Anstellung kirchbergischen Kaminfegers, desgl. von Spielleuten in Ausübung des Kirchweihschutzes sowie durch Beanspruchung persönlichen Vorrangs vor übrigen Ganerben, ferner Belagerung und Überfall einer zum Streifen auf Gaunergesindel ausgeschickten kläg. Patrouille im Köhnschen Wirtshaus zu Gaggstatt.“"11

Es ging also um die gleichen Punkte, wegen derer Comburg und Hohenlohe-Kirchberg auch in Mistlau im Streit lagen - nur dass Hohenlohe-Kirchberg seine rechtliche Position im Fall Mistlau offenbar für stärker hielt als im Fall Gaggstatt.

Am 24. November $1788 \mathrm{kam}$ es dann zu einem ersten Kontakt: Weis besuchte Cunradi in Kirchberg. ${ }^{12}$ Der nahm danach darauf Bezug in einem Schreiben an Weis vom 1. Dezember 1788. Er erkundigte sich zunächst, ob Weis bey der großen Kälte und eingetrettenen Nacht glücklich nach Hauß zurückgekommen sei - wenn ja, so gereiche es ihm zum besonderen Vergnügen. Er habe inzwischen den Fürsten über das Gespräch informiert, und der habe die hohenlohische Bereitschaft zu einer gütlichen Lösung nochmals bestätigt. Nur möge doch Comburg vorher ein schriftliches Verzeichnüß der jenseitig vorgeblichen Beschwerungs Puncte vorlegen, damit die Kirchberger Delegation entsprechend vorbereitet werden könne.

\section{Die Comburger Beschwerden}

Diese Liste traf am 7. Januar in Kirchberg ein. ${ }^{13}$ Weis berichtete darin zunächst, dass er nach der letzten Zusammenkunft zwar spät und erkältet, aber im Übrigen gesund nach Hause gekommen sei. Acht Tage später habe ihn ein starker Katharr überfallen - daher diese verspätete Antwort. Kirchberg müssten zwar eigentlich die Differenz-Punkte wegen Mistlau bekannt sein. Da es der Fürst aber wünsche, wiederhole er hier noch einmal: Comburg fühle sich gekränkt, weil Kirchberg

11 Brunotte / Weber (wie Anm. 7), Nr. 627, S. 441.

12 Der Termin ergibt sich aus dem am 1. Dezember 1788 folgenden Schreiben von Cunradi an Weis (AN 9).

13 AN 10. 
a.) das Recht die Frevel auf Gassen und Straßen zu untersuchen und zu strafen, b.) das Policei und Straifrecht, c.) die Malefiz, d.) die Jurisdiction im Hirtenhau $\beta$ unter Ausschluss des Hochadeligen Ritterstifts für sich beanspruche, auch e.) den Kirchweihschutz unrechtmäßig für sich beanspruche, und f.) speziell diesen in letzter Zeit auch noch auszuweiten versuche. Diese seien - ohne dass er Vollständigkeit beanspruche - die bekannten Punkte. Der Brief schloss mit guten Wünschen für das neue Jahr.

Diese Zusammenstellung war nun sicher nicht das, was sich Kirchberg vorgestellt hatte. Denn die Punkte waren ja auch in Kirchberg bekannt - interessant wäre gewesen, die Comburger Position im Einzelnen zu hören und daraus die Schwachpunkte herausfiltern zu können; dazu hätten diese aber genauer dargestellt werden müssen. Diese Blöße gab sich Weis nicht. Da Comburg sich nicht aus der Deckung wagte, hielt es Kirchberg nicht anders. In seinem Antwortschreiben vom 30. Januar 1789 sagte Cunradi, die Liste von Weis setze ihn in Verlegenheit. Denn in allen diesen Punkten sollten ja nur die Rechte Kirchbergs beschnitten werden; sie seien insofern für Kirchberg überhaupt nicht verhandelbar. Mit Erlaubnis seines Fürsten wäre er aber bereit, zu diesen Punkten (mündlich) die Position Kirchbergs zu erläutern und aus den Akten zu belegen. Er wollte seinerseits zwei Beschwerdepunkte hinzufügen: 1. Das Streifen in Mistlau, das sich Comburg neuerdings anmaße, 2. Den Neugereut-Zehnten, den Comburg für seine Untertanen Kirchberg seit mehreren Jahren vorenthalte.

Beim Streifen geht es um das Recht, zur Aufrechterhaltung der öffentlichen Sicherheit im Dorf Patrouille zu gehen. Nach Kirchberger Ansicht ergab sich das Streifrecht aus der vogteilichen Obrigkeit auf Gassen und Straßen, und deshalb bestand Kirchberg auch darauf, die Streifen ausschließlich durch seine Kräfte durchführen zu lassen. Der Zehnt war grundsätzlich an die jeweilige Herrschaft abzuführen, welcher der Acker gehörte, auf dem geerntet wurde. Für die Comburger Untertanen war das in der Regel Comburg, für die Kirchberger Untertanen Kirchberg. Was passierte nun aber, wenn Wald gerodet und auf dem gerodeten Grund gesät und geerntet wird? Welcher Herrschaft gehört dann der Zehnt? Kirchberg argumentierte, dass es die territoriale Obrigkeit über Mistlau besitze; also gehöre das gerodete Land Kirchberg und der Zehnte war - auch von Comburger Untertanen - an Kirchberg abzuführen. Comburg bestritt die territoriale Oberherrschaft Kirchbergs und beanspruchte auch für das „neu“"14 gerodete Land seiner Untertanen den Zehnten für sich. Diese beiden Punkte wollte also Kirchberg in die Verhandlungen einbezogen haben. 


\section{Schwierig, einen Termin zu finden}

In seinem Antwortschreiben vom 21. März 1789 entschuldigte sich Weis zuerst wieder einmal für eine späte Antwort - teils sei die Verspätung anderen Geschäften, teils ihm zugefallener Unpässlichkeiten geschuldet. Dass er inhaltlich anderer Meinung sei als Cunradi, sei ja klar - aber man werde sehen, wem die Akten Recht gäben. Und natürlich stehe es Kirchberg frei, eigene Punkte in die Verhandlungen einzubringen. Wegen eines Treffens schlug Weis vor, sich frühestens zehn Tage vor Ostern in Cröffelbach ${ }^{15}$ zu treffen, und zwar wegen der Fastenzeit an einem Montag oder Donnerstag. Cröffelbach war als Konferenz-Ort gut gewählt - es liegt von Comburg $10 \mathrm{~km}$ entfernt, von Kirchberg aus $18 \mathrm{~km}$, vor allem aber wurde es von der Postkutsche von Schwäbisch Hall nach Rothenburg von beiden Richtungen her angefahren. Außerdem hatte es ein für einen solchen Zweck geeignetes Wirtshaus. ${ }^{16}$

Cunradi musste in einem Brief an Weis vom 28. März um Aufschub bitten: Sein Sohn heiratet Osterdienstag (hiesiger Stadtschreiber, wie Cunradi nicht ohne väterlichen Stolz hinzufügt), außerdem hielten ihn die sehr gehäuften Berufsgeschäfte ab. Vor Mai sei an die Konferenz nicht zu denken. Er werde sich dann wieder melden.

Es wurde dann August, bis sich wieder etwas tat. Weis meldete sich am 28. August wieder. ${ }^{17}$ Seine Herrschaft wolle in Kürze einen Bericht zur Lage der Dinge. Dem Ort Cröffelbach habe Cunradi ja schon zugestimmt - er schlug vor, sich am übernächsten Tag, samstags, oder am nächsten Montagnachmittag um halb zwei in Cröffelbach zu treffen.

Die Sache verzögerte sich aber weiter. Am 14. September schlug Cunradi den 30. September oder den 1. Oktober vor - und Weis sagte zum 1 . Oktober zu. ${ }^{18}$ Er werde fünf Minuten nach 9 Uhr mit der Postkutsche ankommen und allda [... Cunradi] vergnüglich erwarten. Er bot auch an, das Lokal zu reservieren - zumal ich alle Stunde Gelegenheit dahin habe. Offenbar fuhr damals die Postkutsche von Hall nach Rothenburg im Stundentakt.

\section{Was sind die Streitpunkte?}

Weis nannte folgende Verhandlungspunkte:

a.) das Recht, die Frevel auf Gassen und Straßen zu untersuchen und zu strafen Es wurde damals die niedrige und die hohe Gerichtsbarkeit unterschieden. Die niedere - auch vogteiliche - Gerichtsbarkeit befasste sich mit kleineren Verge-

15 In den Dokumenten finden sich die Schreibweisen Cröffelbach, Cröpfelbach und Cräffelbach. 16 Es dürfte sich um den auch noch heute bestehenden „Goldenen Ochsen“ in Cröffelbach handeln.

17 AN 14.

18 AN 15. 
hen wie Eigentumsdelikten, Schlägereien, Beleidigungen, Störungen der öffentlichen Ordnung und ähnlichem. Davon unterschieden wurde die hohe Gerichtsbarkeit, auch Cent-, Fraisch- oder Blutsgerichtsbarkeit. Sie befasste sich mit Kapitalverbrechen wie Mord und Totschlag. Die Grenze zwischen beiden war nicht ganz fest; meist gehörte zur niederen Gerichtsbarkeit alles, was mit Geldstrafen gesühnt werden konnte.

Was nun die niedere Gerichtsbarkeit betrifft, so waren sich Comburg und Kirchberg darin einig, dass innerhalb der Häuser die jeweilige Herrschaft zuständig war. Strittig war die Gerichtsbarkeit auf Gassen und Straßen, welche Kirchberg für sich beanspruchte. Comburg dagegen wollte für seine Untertanen auch die niedere Gerichtsbarkeit auf Gassen und Straßen ausüben. Die Aufteilung der niederen Gerichtsbarkeit auf zwei Herrschaften bot Anlass zu den ständigen Auseinandersetzungen. Wer war zuständig, wenn ein Comburger und ein Kirchberger Untertan in eine Schlägerei verwickelt waren? Comburg oder Kirchberg? Eigentlich war z.B. Comburg zuständig, wenn im Comburger Wirtshaus zwei Comburger in Streit gerieten. Kompliziert wurde es, wenn der Wirt sie hinauswarf und sie auf der Straße weiter schlägerten. ${ }^{19}$ Nach Kirchberger Auffassung war nun Kirchberg zuständig.

Faktisch sah es so aus, dass in den Fällen, in welchen Kirchberg der Täter auf Gassen und Straßen habhaft wurde, Kirchberg diese aburteilte. In den meisten Fällen war dies so, schon weil Kirchberg viel näher am Tatort war. Von Kirchberg nach Mistlau sind es 2,5 km (eine gute halbe Stunde), von Comburg nach Mistlau rund $25 \mathrm{~km}$, ein Eingreifen also viel schwieriger. Comburg hatte dann jeweils schriftlich protestiert, Kirchberg diesen Protest zurückgewiesen. Damit hatte es sich dann. Da die Straffälle nicht zu häufig waren, konnten beide Seiten mit diesem Zustand offenbar 150 Jahre lang ganz gut leben.

b.) das Policei und Straifrecht

Bevor es zu Gericht ging, musste der Täter erst einmal gefasst werden - und da gab es rasch gravierende Probleme: Was geschah, wenn ein Kirchberger Delinquent sich in ein Comburger Haus flüchtete? Dann war er für die Kirchberger nicht mehr greifbar. Und was geschah, wenn Landstreicher (,liederliches Bettelgesindel") etwas stahlen? Sie waren weder Comburger noch Kirchberger Untertanen. Wer war jetzt zuständig?

c.) die Malefiz

Dabei ging es um Kapitalverbrechen. Kirchberg beanspruchte das Malefizrecht auch über die Comburger Untertanen für sich; Comburg bestritt das.

d.) die Jurisdiction im Hirtenhauß

Die Hirten waren keine Mitglieder der Gemeinde, sondern Auswärtige, welche von der Gemeinde angestellt wurden. Das Hirtenhaus gehörte der Gemeinde, war also weder Comburger noch Kirchberger Eigentum - so ließ sich darüber 
streiten, wer dort die Gerichtsbarkeit ausüben sollte. Symbol der Gerichtsbarkeit über den Gemeindehirten war der Hirtenstab.

e.) der Kirchweihschutz

Die Kirchweih war das wichtigste Dorffest im Jahresverlauf. Die meisten Kirchweihen fanden nach der Ernte im September statt und dauerten von Freitagnachmittag bis Samstagnachmittag. Traditionell wurde viel Wein getrunken. Schlägereien kamen häufig vor, die die Obrigkeit zu verhindern suchte. Außerdem hatte die Herrschaft das Maß zu kontrollieren, nach dem ausgeschenkt wurde, und vergab das Schankmonopol mit entsprechender Abgabe („Bannwein“). Der Kirchweihschutz war also ein wichtiger Anlass für die Herrschaft, Präsenz zu zeigen - und zudem ein einträgliches Geschäft. Im Rahmen seines Anspruchs auf die niedere Gerichtsbarkeit auf Gassen und Straßen beanspruchte Kirchberg auch den Kirchweihschutz und übte ihn aus; Comburg verlangte hier Beteiligung. Von Kirchberger Seite aus kamen noch vier Punkte hinzu. Es ging darum, 1.) dass Comburg neuerdings in Mistlau Streife gehen ließ, 2.) dass es den Neugereut-Zehnten verweigerte, 3.) dass es in Cent- und Fraißfällen der Kirchberger Justiz den Zutritt zu Comburger Häusern verweigerte und Beweisstücke nicht herausgab, 4.) dass es in Einzelfällen Entscheidungen des Kirchberger Consistoriums nicht anerkannte.

Dieser letztere Punkt sollte später noch eine größere Rolle spielen. Hier ging es um Folgendes: Wollten Verwandte zweiten Grades (Vetter und Base) heiraten oder wollte ein(e) Verwitwete(r) vor Ablauf der Trauerfrist von einem Jahr wieder heiraten, war dazu eine Erlaubnis der kirchlichen Verwaltung, des evangelischen Consistoriums, notwendig. Dieser verweigerte das katholische Comburg die Anerkennung.

\section{Das Cröffelbacher Gespräch}

Soweit also die Ausgangssituation für das Gespräch zwischen Weis und Cunradi am Mittwoch, 1. Oktober 1789. Es ist nicht uninteressant, den Gang des Gesprächs im Einzelnen nachzuvollziehen, da man so einen nicht oft überlieferten Einblick in die beiderseitige Argumentation und die Art gewinnt, wie im späten 18. Jahrhundert verhandelt wurde. Ein durchaus zivilisierter Umgang der Parteien und eine Gesprächsführung auf hohem Niveau werden aus dem von Cunradi unmittelbar nach dem Gespräch, am Freitag, 3. Oktober, verfassten Bericht an seinen Fürsten erkennbar. Er schrieb:

Zufolge der zwischen uns genommenen schriftlichen Abrede trafe ich am 1. dieses Vormittags um 9 Uhr in Cräffelbach ein, wo der Herr Geheimrath und Syndicus, Weiß, nur etliche Minuten vorher angekommen war. Dieser, welcher sich bereits in der bestellten oberen Stube befand, kam mir in die untere Stub entgegen, bewillkommte mich auf das höflichste und führte mich bey der Hand in das obere Zimmer, allwo ich auch den H. Bauschreiber von Comburg antraf, der 
sich aber, als er mich allein kommen sahe, sogleich entfernte, so dass ich und Herr Geh.rath ganz allein waren. Wir unterhielten uns anfangs mit allerhand gleichgültigen Gesprächen, biß sich diese auf den Hauptzwek einlenkten.

Offenbar hatte Weis in einem Privatschreiben an Cunradi schon Vorschläge unterbreitet; diese wies Cunradi als unzureichend zurück. Dann ging es zuerst um das Streifrecht. Weis teilte mit, Comburg sei bereit, Kirchberg zuzugestehen, dass dieses weiterhin allein Streifzüge durchführe, wünsche aber jährlich einmal einen gemeinsamen Streifzug. Dies lehnte Cunradi ab. Weis schlug darauf vor, dass die Würzburgische Hußarre, ${ }^{20}$ wenn sie ihren gewöhnlichen Straifzug in Comburg und Allmersband ${ }^{21}$ vornehmen, auch in der jenseitigen Unterthanenhäußeren zu Mistlau Nachsuche auf etwa vorhandenes lüderliches Gesindel thun dürften - auch dies ging Cunradi zu weit. Weis bestand darauf nicht weiter auf einem Streifrecht für Comburg, wollte aber für Comburg vorbehalten, dass die dißseitige, zum Straiffen commandirte Mannschaft nicht in die jenseitige Unterthanenhä̈̈ßer eindringen, sondern nur vor dem Hauß den Besizer fragen, ob kein frembde, verdächtige Leute bey ihm befindlich seyen? da dann der jenseitige Unterthan verbunden seyn solle eine bescheidene und warhafte Antwort darauf zu geben, und wenn sich in der Folge das Gegentheil erfinden würde, so solle er in eine Strafe verfallen seyn. Dies erschien Cunradi akzeptabel.

Was die niedere Gerichtsbarkeit anbelangte, wollte Weis erreichen, dass die Frevel auf Gasse und Straßen von Comburg und Kirchberg gemeinsam untersucht und bestraft werden sollten. Das lehnte Cunradi mit Verweis auf das Kammergerichtsurteil ab. Weis stellte dem die comburgische Interpretation des Kammergerichtsurteils entgegen: Das Gericht habe nur die alleinige Zuständigkeit von Comburg abgelehnt, aber nicht Kirchberg die alleinige Zuständigkeit zuerkannt. Es entspann sich eine Diskussion auf den Linien der Auseinandersetzung beim Kammergericht von 1688 bis 1700 . Interessant ist dann ein Verweis von Weis auf die Erneuerung der Mistlauer Gemeindeordnung von 1611, die damals von Comburg, Kirchberg und Crailsheim gemeinsam vorgenommen worden war. Weis interpretierte dies als Hinweis auf die gemeinsame Zuständigkeit für die niedere Gerichtsbarkeit. Cunradi argumentierte, dabei habe es sich um einen einmaligen Vorgang gehandelt. Weis verwies auf weitere Präzedenzfälle und legte schließlich eine schon fertige Klageschrift auf den Tisch, die man einreichen wolle, wenn es nicht zu einer gütlichen Einigung käme.

20 Comburg besaß nur eine eingeschränkte Selbstverwaltung. Es gehörte zum Fürstbistum Würzburg und wurde von diesem nach außen vertreten. Comburg hatte deshalb auch keine eigenen Soldaten oder Polizei. Eine Polizei in heutigem Sinne existierte noch nicht. Entweder übernahmen am Ende des 18. Jahrhunderts Militär - hier: Husaren - die Streiftätigkeit oder die Einwohner selbst; vgl. Gerhard Fritz: Eine Rotte von allerhandt rauberischem Gesindt. Öffentliche Sicherheit in Südwestdeutschland vom Ende des Dreißigjährigen Krieges bis zum Ende des Alten Reiches (Stuttgarter historische Studien zur Landes- und Wirtschaftsgeschichte 6). Ostfildern 2004, S. 457-646, insbesondere S. 558-622.

21 Heute: Großallmerspann, Ortsteil von Ilshofen. 
$\mathrm{Zu}$ dem von Cunradi angesprochenen Neugereut-Zehnten meinte Weis, im Mistlauer Gemeindebrief von 1569 sei für aufgeteilten Gemeindegrund ausdrücklich Zehntfreiheit beschlossen worden. Cunradi konterte: Damals sei ein neuer Steg über die Jagst notwendig gewesen, und zu diesem Zweck habe man für die damals ausgeteilten Gemeindegrundstücke Zehntfreiheit beschlossen; das gelte selbstverständlich aber nicht für die später ausgeteilten.

Cunradi schlug schließlich vor: Wenn Comburg Kirchberg in der Frage des Neugereut-Zehnten nachgebe, könne Kirchberg in der Frage der fraischlichen Gerichtsbarkeit entgegenkommen - etwa indem man deren Erstreckung auch auf die comburgischen Häuser auf große und schwere Verbrechen einschränke. Diese müssten dann aber genau definiert werden.

Dies fand Anklang bei Weis, der darüber hinaus sogar anbot, Kirchberg den Hirtenstab ganz zu überlassen; nur müsse die Bestellung des Gemeindehirten in der Hand der Gemeinde bleiben. Hinsichtlich der niederen Gerichtsbarkeit schlug Weis vor, dass diese in Kirchberger Hand bleiben solle. Wenn aber ein in Mistlau wohnender Comburger Untertan zu einer Geldstrafe verurteilt würde, sollte Comburg die Hälfte des Geldes erhalten. Bei fremden oder Comburger Untertanen aus anderen Orten solle Kirchberg das ganze Geld behalten dürfen. Geldstrafen, die in Zusammenhang mit der Kirchweih ausgesprochen werden, solle Kirchberg ebenfalls voll behalten dürfen.

Cunradi nahm diese Vorschläge zur Kenntnis und bemerkte noch, dass die Frage der Anerkennung von Consistorial-Angelegenheiten beim nächsten Treffen besprochen werden müsse. Womit die diesmalige Verhandlung beschloßen wurde.

\section{Bewertung des Gesprächs}

Am 7. Oktober 1789 ergänzte Cunradi seinen Bericht mit einem Memorandum für den Fürsten. Zum Ton des Gesprächs bemerkte er noch: Im Hauptwerk hat er (Weis) den vorigen Ton ziemlich herabgestimmt und sich im Umgang ausnehmend freundschaftich erwießen, auch die übertriebene Versicherung gegen mich gethan: dass, wenn er selbst im Hochfürstl. Hohenlohe-Kirchberg. Rath wäre, seine Vorschläge nicht beßer für das hiesig-hochfürstl. Hauße eingerichtet seyn könten.

$\mathrm{Zu}$ den Vorschlägen Comburgs bemerkte er:

1.) Zum Streifen: Hier verliere Kirchberg nichts, da es auch bisher so gehalten worden sei, dass man die Comburgischen Untertanen vor deren Häusern befragt habe, ob sie keine fremden verdächtigen Leute bei sich hätten. Bei Schwerverbrechern habe Kirchberg sowieso das Recht, in die Comburger Häuser einzudringen. Nur habe Weis ausgebeten, dass in solchen Fällen der comburgische Schultheiß in Mistlau um Erlaubnis gebeten werde. Da müsse man ausbedingen, dass die Auslieferung nicht versagt oder verzögert werden dürfe. 
2.) Bedenklicher äußerte sich Cunradi zum zweiten Punkt: der Aufteilung von Geldstrafen zwischen Comburg und Kirchberg, da dieses dem Urteil von 1566 widerspreche. Andrerseits stehe dem das Angebot des Comburger Verzichts auf den Neugereut-Zehnten und den Hirtenstab gegenüber. Vielleicht, so Cunradi, könne man das so regeln, dass die Geldstrafen über 5 Gulden ganz bei Kirchberg verblieben.

3.) Bei den kleineren Verbrechen in Comburger Häusern sei Kirchberg ja willens, diese ganz Comburg zu überlassen. Hier stellte sich die Frage der Abgrenzung. Comburg wollte den einfachen Ehebruch hier eingeschlossen haben. Das war für Cunradi ein Problem, da daraus dann oft eine Ehescheidung entstehe, und die gehöre ins Gebiet der Episcopal- und Kirchenherrschaft, um welche Kirchberg so lange gekämpft habe. ${ }^{22}$ Da müsse noch nachgearbeitet werden.

4.) Und in diesem Zusammenhang müsse Kirchberg auch noch für die Anerkennung von Dispensen in Ehesachen durch Comburg kämpfen.

Cunradi empfahl, den Mistlauer Vergleich erst abzuschließen, wenn auch für Gaggstatt eine Lösung erreicht sei - so habe man immer noch die Möglichkeit, in Mistlau nachzugeben, um den Gaggstatter Prozess loszuwerden.

Der Fürst hatte Bedenken gegen die Anfrage beim Schultheißen vor Auslieferung eines Verdächtigen, da dies doch der Kirchberger hochfraischlichen Obrigkeit widerspreche, und meinte, ob es da nicht besser sei, Comburg eine jährliche Streife in seinen eigenen Häusern zuzugestehen.

Der Handel der Comburger Beteiligung an Geldstrafen gegen Neugereut-Zehnten für Kirchberg leuchtete ihm ein, er vermutete aber, dass Comburg der Beschränkung auf 5 Gulden kaum zustimmen werde, da sie durch Kirchberg leicht unterlaufen werden könne, indem man vermehrt Geldstrafen über 5 Gulden ausspreche. ${ }^{23}$

\section{Die (zweite) Gaggstatter Friedenskonferenz}

\section{Vorbereitungen}

Nach diesem informellen Gespräch musste als nächster Schritt nun eine offizielle Konferenz folgen, um die angedachten Kompromisse in feste Form zu bringen. Bei Kirchberg hatte man aber keine Eile. Weis fragte deshalb Anfang April 1790 in Kirchberg nach, ob man noch Interesse an einem Vergleich habe oder es doch lieber auf eine gerichtliche Auseinandersetzung ankommen lassen wolle. Cunradi beeilte sich, am 15. April zu versichern, dass man nach wie vor am Vergleich interessiert sei, es sei aber wegen anderer Geschäfte alles liegen geblie-

22 Vgl. dazu Ulrich Fröhner: Der Mistlauer Taufstreit 1747-1754. In: WFr 97 (2013), S. 177202.

23 AN 19; undatierte Aktennotiz des Fürsten. 
ben. Außerdem habe man einen neuen Assessor, der sich erst einarbeiten müsse. Mindestens sechs Wochen werde man noch benötigen zu einem conferenzialischen Zusammentritt. ${ }^{24}$

Den Aufschub nutzte Kirchberg dazu, das Seniorat des Hauses Hohenlohe einzuschalten, das das Gesamthaus nach außen vertrat. Für dieses gab Geheimrat Zeller aus Langenburg am 29. Mai 1790 seine Zustimmung zum vorgesehenen Vorgehen. ${ }^{25}$ Aber die neue Konferenz verzögerte sich bis 1791. Zuvor hatte es offenbar neue Irritationen wegen Gaggstatt gegeben, ${ }^{26}$ Hohenlohe wiederholte aber sein Interesse an einer Konferenz und schlug wieder ein Treffen in Cröffelbach vor. Weis war einverstanden. ${ }^{27}$ Auch Zeller sollte hinzugezogen werden. ${ }^{28}$ Man fasste den März 1791 ins Auge. Wohl weil nun doch eine größere Anzahl Personen hinzugezogen werden musste, brachte Kirchberg als Konferenzort Gaggstatt ins Spiel. Dort war es viele Jahre zuvor gelungen, die Konfessionsstreitigkeiten mit Comburg wegen Mistlau beizulegen. ${ }^{29}$ Weis teilte mit, er werde am 1. März, früh gegen 9 Uhr eintreffen; das Mittagessen betreffend könne Kirchberg das erste ausrichten, danach könne man sich abwechseln. ${ }^{30}$

Auch das Äußere einer solchen Konferenz erforderte einige Vorbereitung. Cunradi fragte beim Fürsten nach, ob das zu gebende tractement (Bewirtung) von Hof aus verlegt oder aber dem Kochendörfer Pachtwirth zur Besorgung übergeben werden solle? Im letzteren Fall werde der ja noch einen Koch oder eine Köchin bestellen müssen, was zu zusätzlichen Kosten führen würde. Aus seiner Sicht wäre es besser, einen Koch und die benötigten Viktualien vom Hof zu schicken, wobey überdieß nach dem Vorgang von 1754 ein Liberay [Livree]-Bedienter zum Tischdecken und Aufwarten während der Mahlzeit nöthig seyn wird.

Der Fürst war einverstanden und wollte von seinen Bedienten den Schramm und den Rösch mitschicken - letzterer sei nicht nur zum Schreiben, sondern auch zum Tischdecken und Servieren gut zu gebrauchen. Dann fragte der Fürst noch, ob auch der Registrator nothwendig gleich dabey sein muss? meiner Vermuthung nach mögte vor erst mehr gesprochen (welches er eben nicht zu hören braucht) als geschrieben werden.

Für die Konferenz wurde am 25. Februar 1791 eine Vollmacht für Zeller und Cunradi ausgestellt. Die Konferenz begann dann am Dienstag, 1. März 1791, 9 Uhr. Geplant war, zwei Tage zusammenzusitzen - es wurden dann tatsächlich

30 AN 28. So war es auch bei der ersten Konferenz gehalten worden. 
vier. Dienstag und Mittwoch tagte man in Gaggstatt, am Donnerstag in der „Krone“ zu Kirchberg, am Freitag noch einmal in Cröffelbach. ${ }^{31}$

\section{Die Konferenz}

Als Verhandelnde anwesend waren zunächst: für Kirchberg der Geheime Hofrat Zeller und Hofrat Cunradi, für Comburg der Syndikus Weis und der Kanzlei-Sekretär Wolf. Am 2. März nahm anstelle von Wolf der Kanzleirat Potschka von Comburg teil, am 3. März zog Kirchberg noch den Rat und Amtmann Schuster bei. Die Hilfskräfte - Koch, Diener, Schreiber - sind natürlich nicht namentlich genannt. $^{32}$

Das 23 Blätter umfassende Protokoll beeindruckt durch das erledigte Arbeitspensum. Neben den in der Vorbereitung angesprochenen Punkten ging es noch um eine Menge anderer Dinge, die wegen Mistlau zu erledigen waren - wie die Förderung des Klee-Anbaus, die Anschaffung eines Wagens durch die Dorfgemeinde oder die Verhinderung des Verkaufs von Getreide nach auswärts bei einer Teuerung. Zu den Hauptpunkten wurde vereinbart:

1. Die niedere Gerichtsbarkeit (Frevel auf Gassen und Straßen) sollte für Hohenloher und Auswärtige von Kirchberg allein ausgeübt werden. Bei Streitigkeiten, in die sowohl Hohenloher als auch Comburger Bürger verwickelt sind, durfte Hohenlohe das Verfahren durchführen, musste aber das Protokoll an Comburg schicken und die Hälfte der Strafe an Comburg abführen (Comburg hatte sich also tatsächlich nicht auf eine Obergrenze bei der abzuführenden Geldstrafe eingelassen). So sollte es auch gehalten werden, wenn es allein um Comburger Untertanen ging. Würden diese auf frischer Tat ertappt, so durfte Hohenlohe sie auch sofort festnehmen; im anderen Fall musste es Auslieferung bei Comburg beantragen und Comburg diese gewähren.

2. Der Hirtenstab: Der Hirt sollte weiterhin von der Gemeinde angestellt werden, die Gerichtsbarkeit über den Hirten ganz bei Kirchberg liegen.

3. Die hohe Gerichtsbarkeit sollte allein von Kirchberg ausgeübt werden. Da die Abgrenzung von hoher und niederer Gerichtsbarkeit nirgends festgelegt sei, wurde ein detaillierter Katalog erstellt über die Delikte, die zur hohen Gerichtsbarkeit gehörten. In diesen Katalog war auch der Ehebruch aufgenommen; es wurden aber Höchststrafen dafür festgesetzt. Genau geregelt war, wie Kirchberg

31 Schreibkalender des Fürsten Christian Friedrich Carl vom Jahr 1791, Einträge vom 2. bis 4. März. HZA Ki 100 B 85. Weis und Cunradi waren am Donnerstag zur fürstlichen Tafel eingeladen. 32 Es existiert in AN 24 ein Beleg über Ausgaben im Wirtshaus in Gaggstadt, der im Herbst 1790 datiert ist. Demnach haben Mundkoch Junker, Cammerschreiber Schramm, Copist Rösch, Kutscher Häfner und Philipp Breitner bey Gelegenheit der Conferenz mit Comburg in Gaggstatt, bey dem Wirth Röhn daselbst über Mittag verzehrt und bezahlt jeweils $31 \mathrm{kr}$. Es kann sich eigentlich nur um die Konferenz im März 1791 oder die zweite im September 1791 gehandelt haben. Die Besetzung dürfte in beiden Fällen gleich gewesen sein. 
bei der Festnahme eines Verdächtigen zu verfahren habe. Geschah dies in einem comburgischen Haus, musste Kirchberg dies vorher beim Comburger Schultheißen in Mistlau anmelden; falls dieser nicht da, oder mit dem Delinquenten verwandt war, beim ältesten Comburger Untertan. Falls diese oder der Delinquent Schwierigkeiten machten, durfte Kirchberg letzteren auch mit Gewalt aus einem comburgischen Haus herausholen. Im Übrigen hatte Comburg das Recht, bei einem Verhör des Delinquenten anwesend zu sein.

Mit der Klärung dieser Punkte war der erste Tag angefüllt. Am zweiten wurde zunächst ein Geldstrafenkatalog bei Fällen von Inzest erstellt. Dann ging es um 4. Das Streifrecht. Dieses sollte wie bisher Kirchberg zustehen. Falls die Streife in einem comburgischen Haus etwas Verdächtiges vermute, sei wie im Punkt 3 zu verfahren, im Fall aber blosse Vagabunden und Bettler sich in einem Comburgischen Hauße enthielten, so wären selbige durch den Comburgischen Schultheißen aus dem Hauße und der Hofreithe zu schaffen und entweder der anweesenden Kirchberger Patrouille oder dem Kirchberg. Schultheißen zu Mistlau auf der Straße zur Captur [Gefangennahme], oder ferneren Fortschaffung zu überlassen.

5. Der Kirchweihschutz: Das Recht des Aneichens und Kannen-Angießens sollte Hohenlohe künftig ohne Widerspruch Comburgs auch im Comburger Wirtshaus und in der Mühle zustehen, allerdings ohne dass dies ein Präzedenzfall auch für andere Ortschaften sei. Über den Bannwein konnte man sich zunächst nicht einigen.

6. Bei Trauerfällen im Kaiserhaus solle Kirchberg wie bisher auch das Trauergeläut anordnen und Verbot von Musik und Tanz verfügen, was dann auch von Comburg für seine Häuser übernommen würde. Bei Trauerfällen im kirchbergischen Fürstenhaus müsste Kirchberg Comburg ersuchen, auch für seine Untertanen ein Verbot von Musik und Tanz auszusprechen; Comburg sagte zu, einem solchen Gesuch zu entsprechen.

7. Musik bei Hochzeiten: Bei Hochzeiten Comburgischer Untertanen soll zum Aufspielen mindestens ein Kirchberger Spielmann hinzugezogen werden. ${ }^{33}$

Mit diesen Festlegungen war der zweite Tag ausgefüllt. Am dritten Tag musste zuerst noch die Frage des Bannweins geklärt werden. Üblicherweise durfte an Kirchweih nur der Bannwein ausgeschenkt werden - das ist Wein, der von der Herrschaft an die Gemeinde geliefert wurde. Kirchberg konnte den beiden Comburger Wirten nicht verbieten, während der Kirchweih Wein auszuschenken. Man kam überein, dass es während der Kirchweih den comburgischen Untertanen nicht erlaubt sei, Wein aus dem comburgischen Wirtshaus zu holen, auch dürften die Wirte keinen Wein über die Gasse schicken. Das bedeutete im Um-

33 Für das Aufspielen bei Hochzeiten und Kirchweihen beanspruchten die Landesherren das Monopol. In Hohenlohe-Kirchberg war es damals so, dass der Fürst das Recht zum Aufspielen gegen Pacht vergab. Konnte der Spielmann bei mehr Gelegenheiten aufspielen, konnte der Fürst auch mehr Pacht verlangen. Ingeborg Wesser: Musikgeschichte der Hohenlohischen Residenzstadt Kirchberg von der Mitte des 17. bis zum Beginn des 19. Jahrhunderts. Stuttgart und Weimar 2001. 
kehrschluss: Im comburgischen Wirtshaus und in der Mühle durfte auch während der Kirchweih eigener Wein ausgeschenkt und getrunken werden - aber eben nicht außerhalb. Bei dieser Gelegenheit einigte man sich auch auf Maßnahmen gegen das übermäßige Trinken bei Gemeindezechen.

8. Der Neugereut-Zehnt: Comburg war (nach langer Diskussion) bereit, diesen Kirchberg zuzugestehen; nur sollte der Kleeanbau auf neu unter den Pflug genommenen Flächen zehntfrei bleiben.

9. Dispense in Ehesachen: Dispense wegen Eheschließung in der Trauerzeit und unter Verwandten durfte Kirchberg allein erteilen. Bei Ehestreitigkeiten sollte der Gaggstatter Pfarrer einen Schlichtungsversuch unternehmen; falls das nicht hülfe, das Consistorium von Kirchberg ein Urteil sprechen. Die Ausführung des Urteils sollte aber durch Comburg erfolgen.

10. Formalien: Am Schluss des Tages hatte man noch eine Rangstreitigkeit erledigt: Bei Sitzungen in Sachen Mistlau sollte Kirchberg den Vorsitz haben, auch den Vorrang bei der Sitzordnung und bei der Unterschrift unter Vereinbarungen; bei der Führung des Protokolls werde man sich abwechseln.

Das war es dann für den dritten Tag. Am vierten blieb noch ein Kirchberger Problem zu erledigen: Bei der Hungersnot Anfang der 1770er Jahre hatte Kirchberg ein Ausfuhr-Verbot für Getreide verhängt. Die Mistlauer Bauern aber hatten (natürlich zu höheren Preisen!) Korn an den Comburger Müller in Mistlau verkauft, der es dann ausgeführt hatte. Comburg sagte - ohne rechtliche Verpflichtung zu, in solchen Fällen in Zukunft einzuschreiten. Dann beschloss man noch, dass die Gemeinde einen Wagen anschaffen und dieser den ärmeren Gemeindebauern bei den gemeinsam zu verrichtenden Arbeiten zur Verfügung gestellt werden sollte.

Soweit die inhaltlichen Festlegungen. Es muss am Schluss der Konferenz - wie bei solchen Gelegenheiten häufig - eine fast euphorische Stimmung unter den Delegierten geherrscht haben. Denn sie beschlossen noch:

a.) in Zeit von 8 Tagen sollten die bey den Protocollen noch abgängige Vollmachten beyderseitiger Deputationen gegeneinander ausgewechselt, ${ }^{34}$

b.) die erhoffende Ratification beyderseits der Höchst $t^{35}$ und Hoher Committenten in Zeit von 6 Wochen einander mitgetheilt, und

c.) nach erfolgter höchst- und hoher Ratification die verabredeten und verglichenen Punkte in einen förmlichen Rece $\beta$ [Vertrag] gebracht, derselbe hierauf gemeinschaftlich ajouhtiret, und nach vorheriger höchst- und hoher Unterfertigung gegeneinander ausgewechselt werden; wobey

34 Comburg hatte es - wie auch bei der ersten Gaggstatter Konferenz - mit den Vollmachten nicht richten können; deshalb hatte man sich darauf geeinigt, diesen Punkt für beide Delegationen auf später zu verschieben.

35 Dem Fürstentum Hohenlohe kam das Prädikat „höchst“ zu, dem Ritterstift Comburg nur „hoch“. 
d.) Comburg anbot, auf den Freiherrn von Seckendorff zu Erkenbrechtshausen zuzugehen und ihn zum Beitritt zu bewegen - dieser hatte auch einen Untertan ${ }^{36}$ in Mistlau.

Nun wurde das Protokoll von Zeller und Cunradi für Hohenlohe und Weis und Potschka für Comburg unterschrieben - dann durften alle ins wohlverdiente Wochenende fahren.

\section{Ratifikation und Nichtratifikation}

Fürst Christian Friedrich Carl zu Hohenlohe-Kirchberg muss sehr gespannt auf das Ergebnis der Konferenz gewesen sein. Jedenfalls las er das Protokoll noch am Samstag, dem 5. März 1791 und genehmigte es ohne Anstände. Damit war die Vereinbarung von Seiten Kirchbergs ratifiziert.

Nicht so auf Seiten Comburgs. In einem Privatbrief von Weis an Cunradi Hochgeehrter, liebster Freund! - man war sich in Gaggstatt auch menschlich näher gekommen - gratulierte dieser Cunradi zunächst zur Ratifikation durch den Fürsten und fuhr dann fort: Nicht so glücklich bin ich meiner Seits, indeme sich schon solche Schwürigkeiten erheben, dass ich veranlasst worden, gestern noch den dritten Expressen nach Wirzburg ${ }^{37}$ zu spediren, der heute frühe abgereist ist. Er werde alles aufbieten, dass es nicht zu inhaltlichen Änderungen komme, sondern es bei der Modifikation gewisser Ausdrücke bleibe. Wegen Erkenbrechtshausen hatte sich Kirchberg offenbar besorgt gezeigt (wie sich später herausstellen sollte, nicht ohne Grund). Weis hoffte, dass von dorther keine Bedenklichkeiten gemacht werden. Schließlich bat Weis, gegenwärtigen Bericht nicht ad Acta kommen zu lassen, worüber ich auf Ihre mir bekannte Redlichkeit zehle.

Weis hatte sich in dem letzten Punkt getäuscht. Nicht nur, dass Cunradi den Brief zu den Akten gab, wo er bis heute liegt, er reichte ihn auch an den Fürsten und Zeller weiter. Beide rätselten, ob Weis bei den Widerständen auf Seiten Comburgs bzw. Würzburgs nicht übertrieb. Der Fürst meint: Vermutlich sucht er hier ein größeres Meritum [Verdienst] sich dadurch zu machen, wenn er sagt, wie viele Mühe er hätte anwenden müßen um seine H(erren) und Oberen zur Einwilligung zu disponieren. Und Zeller vermutete, Weis schütze hier Würzburg vor, um einige Formulierungen aus dem Vertrag herauszubekommen, die ihm selbst anstößig seien.

Am 4. Mai 1791 wurde Weis in einem neuen Privatbrief an Cunradi konkreter. ${ }^{38}$ In einem diesmal wieder offiziellen Brief an Cunradi teilte er mit, dass Comburg

36 Untertan bezieht sich jeweils auf die ganze Familie inklusive Knechten und Mägden.

37 Comburg war nicht reichsunmittelbar, sondern wurde nach außen vom Fürstbistum Würzburg vertreten. Verträge mit anderen Staaten mussten deshalb von Würzburg genehmigt werden.

38 AN 36. 
erst auf der nächsten Sitzung am 20./21. Juni über den Vergleich beraten werde. Unter der Hand habe er aber schon erfahren, was Comburg an zusätzlichen Forderungen stellen wolle:

- Zum einen ging es um einige Formulierungen, bei denen Comburg seine Souveränitätsrechte zu sehr beschnitten sah oder für die Zukunft eine solche Beschneidung fürchtete. ${ }^{39}$

- Zum andern sollte die Strafe bei Inzest auf ein Drittel herabgesetzt werden.

- Zum Dritten sollte bei Trauerfällen Gegenrecht herrschen: Es sollten also auch Lustbarkeiten der hohenlohischen Untertanen verboten werden, wenn ein Dekan von Comburg starb.

Weis empfahl, sich auf diese Forderungen einzulassen, da sich einige der Herrn in Comburg mit der Ratifikation des Vergleichs ohnehin sehr schwer tun würden. Am 31. Mai 1791 berieten Zeller, Cunradi und Taurinus, wie mit den Comburger Änderungswünschen umzugehen sei. ${ }^{40}$ Sie beschlossen: Cunradi solle in einem Privatschreiben an Weis mitteilen:

1. Zu den Punkten, bei denen es nur um rechtliche Vorbehalte bei den Formulierungen gehe, könne Kirchberg in den meisten Fällen zustimmen.

2. Auch was die Herabsetzung der Strafen bei Inzest anging, war Kirchberg um des lieben Friedens willen bereit mitzumachen.

3. Was das Verbot von Lustbarkeiten beim Ableben eines Comburger Dekans betraf, sei solches bisher nie von comburgischer Seite verlangt und nie Gegenstand von Verhandlungen gewesen, deshalb könne dem auch nicht zugestimmt werden.

Es folgte weiterer Briefwechsel zwischen Weis und Cunradi, an dessen Ende schließlich zwei Punkte offen blieben. ${ }^{41}$

1. Zum einen war es die Frage des Verbots von Lustbarkeiten bei Comburger Trauerfällen.

2. Comburg bestand auf einer Generalklausel am Schluss des Vertrags: dass nämlich in allen im Vertrag nicht genannten Punkten die beiderseitigen Ganerbiats-Gerechtsame bestehen bleiben sollten.

Weis griff außerdem zu einem ungewöhnlichen Mittel: In einem vertraulichen Privatbrief $^{42}$ an den Fürsten appellierte er an diesen, die beiden ohnschädliche Desideria gnädigst zu bewilligen, da ich

a) wohl ersichtlich im ganzen Vergleichswesen mich sehr nachgiebig [...] angelassen, b) in der Gaggstatter Sache alles mögliche thun werde, endlich mir c) an den Punktis quaest. [den in Frage stehenden Punkten] um so mehr gelegen, da

39 Ein Beispiel: statt hohe und fraischliche Obrigkeit, die Kirchberg zugestanden wird, solle es heißen: hohe fraischliche Obrigkeit.

$40 \quad$ AN 37.

41 AN 38 Weis an Cunradi am 15. Juni 1791.

42 AN 39. Dieser Brief enthält kein Datum. Lag er dem Brief an Cunradi vom 15. Juni bei? 
diese durch das wichtige votum - jedoch in engsten Vertrauen gesagt - Sr. Excellenz Herrn von Fechenbach ${ }^{43}$ nachdrücklichst erinnert worden.

Offenbar fruchtete dieser Appell nicht. Nachdem sich Zeller am 16. Juni 1791 schon dafür ausgesprochen hatte, in der Trauerfrage hart zu bleiben (beim Übrigen könne man nachgeben), antwortete Cunradi am 18. Juni in einem offiziellen Schreiben an Weis: ${ }^{44}$

In der Trauerfrage könne Kirchberg nicht nachgeben, da in Gaggstatt vereinbart worden sei, es bei allem, was nicht Gegenstand der Verhandlungen gewesen sei, beim Alten zu lassen.

Kirchberg argumentierte hier also rein formal; dazu auf etwas wackeligem Boden: Denn natürlich war die Trauerfrage in Gaggstatt zur Sprache gekommen, wenn auch nur in umgekehrter Richtung, nämlich in Bezug auf das Verhalten der Comburgischen Bürger bei Kirchberger Trauerfällen.

Was den zweiten Punkt betraf, nämlich die Generalklausel daß in allübrigen, in diesem Vertrag nicht benannten Puncten die beyderseitige Ganerbiats-Gerechtsame aufrecht bestehen sollen sei man bereit, diese hinzunehmen. Sie werden hieraus die dießseitige aufrichtige Neigung sich auf in alle nur mögliche Art willfährig zu erweißen auf das neue zu ersehen belieben schrieb Cunradi am Schluss seines Briefes.

Die Comburger Kapitel-Konferenz am 20./21. Juni scheint entweder nicht stattgefunden oder in der Mistlauer Angelegenheit zu keinem Erfolg geführt zu haben. Am 2. Juli schrieb Weis wieder einmal an Cunradi: ${ }^{45}$ Er sehe überhaupt keinen Grund, warum man sich in der Trauerfrage nicht einigen können solle und warum dadurch der ganze Mistlauer Vertrag, wo aus allen Zeilen der jenseitige Vortheil sichtbar hervorleuchtet, aufgehalten werden sollte. Er zweifle nicht daran, dass man sich auch in diesem letzten Punkt noch einig werde und wünsche sehr, dass dies so pünktlich geschehe, dass Comburg bei der ersten Kommunikation zustimmen könne.

Offenbar hatte Weis auch damit keinen Erfolg. Er sah sich deshalb veranlasst, um den 5. August das Comburger Begehren etwas abzuschwächen: ${ }^{46}$ Die Trauerfrist sollte nur acht Tage sein, und Kirchweih- und Markt-Tage davon ausgenommen werden. Weis fürchtete, dass wegen dieser Kleinigkeit das ganze Vergleichs Weeßen mögte [...] rückwärts gehen. Damit hatte Weis Recht, wie sich zeigen sollte.

Am 15. August wies Cunradi das Anliegen Comburgs wieder mit formalen Gründen zurück. Weis antwortete am 23. August 1791 teils zornig, teils resigniert. ${ }^{47}$ In dieser Situation wurde auch wieder Zeller um Rat gefragt. Er schrieb

47 AN 44; vgl. unten Anhang 3 dieses Beitrags. 
am 29. August, ${ }^{48}$ einerseits könne man in der Trauerfrage nicht nachgeben; andererseits dürfe man wegen Mistlau die Gaggstatter Vergleichsverhandlungen nicht gefährden. Er stimmte daher der Idee Kirchbergs zu, diesen Punkt auf die Tagesordnung der nächsten Vergleichsverhandlung wegen Gaggstatt zu setzen. Comburg seinerseits roch den Braten und teilte mit, es wolle über Gaggstatt erst reden, wenn der Mistlauer Vergleich abgeschlossen sei. Weis erklärte sich deshalb vorerst nur zu einem informellen Gespräch bereit, ${ }^{49}$ das am 13. September wieder in Gaggstatt abgehalten wurde. Es ging im Wesentlichen um die Gaggstatter Angelegenheit, es kam aber auch Mistlau zur Sprache. Eingeladen waren neben den Vertretern von Comburg und Kirchberg auch Amtmann Wiedmann ${ }^{50}$ von Seckendorff-Erkenbrechtshausen und Amtmann Schüz von Crailsheim-Hornberg.

Auf der Konferenz, die vom 13. bis zum 16. September dauerte, spielte Mistlau nur eine Nebenrolle. Es ging um Gaggstatt. Bezüglich Mistlaus, einigte man sich in der Trauerfrage folgendermaßen: Da Kirchberg sich nicht darauf einlassen wollte, seinen Untertanen Staatstrauer beim Tod eines Comburger Dekans zu verordnen, zog Comburg seine Zusage zurück, dies bei einem Kirchberger Trauerfall bei seinen Untertanen zu tun.

Nun aber machte Seckendorff Probleme. Amtmann Wiedmann erklärte, seine Herrschaft werde dem Vergleich nur beitreten, wenn

1. Kirchberg seine Forderung zurückziehe, dass zum Aufspielen bei Hochzeiten immer wenigstens ein Kirchberger Spielmann beteiligt werde,

2. Seckendorff das Dispensationsrecht über seinen Untertanen selbst ausüben dürfe,

3. Kirchberg nicht auf dem Entscheidungsrecht seines Consistoriums bei Ehestreitigkeiten bestehe.

Kirchberg nahm diese Forderungen zur Kenntnis; wies aber gleich darauf hin, dass Punkt 2 und 3 gegen das Jus Episcopale Kirchbergs verstoßen würden weshalb Kirchberg hier unmöglich nachgeben könne.

Damit beließ man es am ersten Tag. Auf das Mistlauer Problem kam man am 16. September wieder zurück. ${ }^{51}$ Kirchberg nahm seine Forderung zurück, dass bei Hochzeiten wenigstens ein Kirchberger Spielmann mit aufspielen solle.

Zu Punkt 2 und 3 der Seckendorffischen Forderung einigte man sich, dass das Vorgehen, das mit Comburg vereinbart wurde, auch auf den seckendorffischen Untertanen ausgedehnt werden solle. Comburg hatte - als Teil des Gaggstatter Handels - nun doch wieder zugestimmt, bei Trauerfällen im Kirchberger Fürstenhaus Lustbarkeiten bei seinen Untertanen zu verbieten, ohne das Gleiche von

48 AN 45.

49 AN 46.

50 Derselbe, der in Kirchberg Assessor war? Die Vornamen stimmen nicht überein - der Assessor in Kirchberg hieß Johann Friedrich Wiedmann, der Seckendorffische Amtmann Johann Michael Wiedmann.

51 AN 53 
Kirchberg zu verlangen. Da anzunehmen sei, dass das Protokoll nun von allen Seiten ratifiziert würde, beschloss man gleich, dass es in die Form eines Recesses - eines förmlichen Verhandlungsergebnisses - gebracht werden solle.

Aber wieder einmal hatten die Deputierten sich geirrt. Comburg hatte - natürlich? - wieder Sonderwünsche. ${ }^{52}$ Dort, wo vom Jus Episcopale Kirchbergs die Rede ist, sollte eingefügt werden: deme aber das RitterStift Comburg und Cons. contradiciren [widersprechen]. Und auch bei den anderen beiden Punkten müsse ein Vorbehalt eingefügt werden.

Für den Donnerstag nach der Konferenz lud übrigens der Dekan die Herren Cunradi, Zeller und Taurinus zu einer geringen Mittagssuppe ein - eine Einladung, die Zeller nicht nur aus Termingründen ablehnt, sondern auch, weil die ganze Tour bey gegenwärtiger Jahreszeit und Witterung mit allzu vieler Beschwerlichkeit von hier aus verknüpft ist. Das schrieb er natürlich nur nach Kirchberg - und wünschte Cunradi und Taurinus viel Vergnügen bei ihrer Tour..$^{53}$

Die Protokolle der Verhandlungen zu Gaggstatt und Mistlau hatte offenbar Zeller mit nach Langenburg genommen. Er schickte sie am 23. September Cunradi zur Einsicht und Prüfung und bat ihn, dem Copisten Rösch einzuschärfen, bei der Reinschrift alle Aufmerksamkeit auf die Rechtschreibung anzuwenden, damit nicht wieder solche Fehler wie im (ersten) Mistlauer Protokoll vorkämen. Kopien sollten unverzüglich zu Weis, nach Erkenbrechtshausen und nach Hornberg geschickt werden; dann könne man einen Tag zum letzten Abgleich und zur Unterschrift vereinbaren. ${ }^{54}$

Der Fürst war auf Reisen. Cunradi schickte ihm am 26. September die Protokolle zu, bat aber, sie gleich zurückzuerhalten, damit er sie abschreiben lassen und an die anderen Beteiligten weitergeben könne. Der Fürst schickte ihm seinen Brief zurück mit der Randbemerkung: um diese [Besorgungen] nicht aufzuhalten, habe ich noch gestern nachts sämtl. Schriften mit dem Wunsche durchlesen, dass die übrige Interessenten keine weitere Einwendungen gegen den Inhalt derselben mehr machen mögten..$^{55}$

Wie man sieht hatte der Fürst allmählich genug von der Sache. Nun gab es eine kleine Pause. Weis hatte mit den Festlichkeiten zum Namenstag des Dekans zu tun. Deshalb konnte er sich erst am 9. Oktober 1791 bei Cunradi für die Übersendung des Protokolls bedanken ${ }^{56}$ und - wieder! - neue Änderungswünsche Comburgs mitschicken. Sie scheinen aber nur Gaggstatt, nicht Mistlau zu betreffen. Der Fürst gab das Schreiben zur Beantwortung an Zeller weiter. ${ }^{57}$

Am 26. November schrieb Weis an Cunradi, nun sei das Vergleichs-Manuskript fertig geworden und er schicke es ihm hiermit, nicht ohne auszudrücken dass er 
sich über die Sophicismen [Spitzfindigkeiten] geärgert habe, durch die die Sache so in die Länge gezogen wurde. ${ }^{58}$

\section{Eine lange Pause}

Nun folgte eine lange Pause. Erst 1799 fragte Comburg bei Kirchberg an, ob dieses ein Interesse daran habe, die Verhandlungen zu einem Abschluss zu bringen. Was ist die Ursache für die lange Pause? Hatten alle Seiten die Lust verloren? Waren sie zu sehr mit anderen Dingen beschäftigt? Immerhin tobte in Frankreich die Revolution und sollte bald in kriegerischer Form auf Deutschland übergreifen. Eine Rolle hat sicher gespielt, dass die beiden Hauptakteure, Weis und Cunradi, in dieser Zeit gestorben sind. Es existiert ein Briefentwurf Comburgs aus dem Jahr 1796, in dem darauf Bezug genommen wird. ${ }^{59}$ Allem Anschein nach ist dieser Brief nie abgeschickt worden. ${ }^{60}$ Cunradi war am 6. Januar 1795 im Alter von fast 71 Jahren gestorben. Über den Tod des Licentiaten Weis war nichts in Erfahrung zu bringen. An die Stelle Cunradis trat der Geheime Hofrat Gottfried Ludwig Knapp; Assessor war jetzt Friedrich Ernst Diezel. In Comburg war Kanzleirat Frick an die Stelle von Weis getreten. Nur der Fürst war immer noch Christian Friedrich Carl - er sollte 90 Jahre alt werden! Und auch auf der Comburg regierte zusammen mit dem Kapitel weiterhin Dekan Johann Godefrid von Greiffenclau.

Kanzleirat Frick stellte sich die Aufgabe, die eingeschlafenen Verhandlungen wieder in Gang zu bringen. Er schreibt deshalb am 13. September 1799 an Knapp. Der antwortete schnell, bat aber um Aufschub: Grundsätzlich sei man nach wie vor an einer Klärung interessiert - aber die personellen Veränderungen in der Kanzlei, die dringenden Aufgaben durch die Kriegszeiten, und die komplizierte Materie, die eine längere Einarbeitung erfordere - all das lasse es nicht zu, sich vor dem Beginn des nächsten Jahres mit der Sache zu befassen. ${ }^{61}$

Im Gegensatz zu den Kirchberger Akten enthalten die Comburger Akten selten etwas über die interne Kommunikation auf Comburger Seite. Das ist hier ausnahmsweise der Fall. Syndicus Licentiat Potschka schreibt am 19. Oktober 1799 an Frick: Kirchberg sei mit Preußen ${ }^{62}$ in Verhandlung, dem an Allmerspann und Gaggstadt gelegen sei - so will auf baldigste Vollendung des Vergleichs wegen

58 AN 62; vgl. Anhang 4.

59 StA Ludwigsburg B 375 L Bü 112. Diese Akte enthält den Comburger Bestand zum Briefwechsel in dieser Sache aus den Jahren 1796 bis 1801.

60 Es fehlt ein Datum beim Absendevermerk; in den Kirchberger Akten findet sich der Brief nicht; und es gibt weder in den Kirchberger noch in den Comburger Akten eine Antwort darauf.

61 Brief vom 30. September 1799, StA Ludwigsburg B 375 L Bü 112.

62 Die Markgrafschaft Ansbach war inzwischen Preußen eingegliedert worden. 
Gaggstadt und Mistlau ohne Verlängerung allenfalls binnen 4 Wochen zu bestehen seyn. ${ }^{63}$

Ganz so grimmig sah es Frick (oder sein Vorgesetzter) nicht. Im nächsten Brief an Knapp vom 23. Oktober äußerte er zunächst Freude darüber, dass auch Kirchberg an einer Erledigung interessiert sei, meinte allerdings, es wäre angenehmer gewesen, dieses Jahr noch Hand an das Geschäft anzulegen: Im ersten Quartal des nächsten Jahres stehe wieder die Hauptversammlung des Kapitels an und daraus könnten sich neue Schwierigkeiten ergeben. Um die Sache zu beschleunigen, werde er aber einen Vertragsentwurf erstellen und der anderen Seite zur Prüfung zustellen. ${ }^{64}$

Wie so viele vor ihm, hat sich auch Frick in diesem Punkt getäuscht, obgleich er erstaunlich schnell mit seinem Vertragsentwurf fertig war. Er schickte ihn am 10. November 1799 an den Dekan zusammen mit einem langen Rechtfertigungsbrief, in dem er zu den für Comburg heiklen Punkten Stellung nahm und um Überprüfung und Billigung bat. ${ }^{65}$

Erst am 17. April 1800 konnte Frick eine Kopie seines Entwurfes an Kirchberg schicken. Sein eigenes Original schickte er gleichzeitig an den Amtmann Diezel in Erkenbrechtshausen mit der Bitte, dies selbst zu kopieren und dann schnell zurückzugeben.

In seinem Begleitschreiben schlug Frick ein Treffen in Großallmerspann vor, um noch einige Details des Entwurfs zu besprechen. Vor allem ging es darum, ob man die in den Briefen zwischen Weis und Cunradi vom 6. und 18. Juni 1791 besprochenen Punkte nicht in das Protokoll aufnehmen solle. Frick hielt dies für nötig - ein Grundsatz, der bis heute gilt: Für die Auslegung eines Vertrages ist seine Vorgeschichte wichtig.

Knapp brauchte einige Zeit, um den Entwurf Fricks durchzuarbeiten. Am 5. Juli legte er seinem Fürsten dazu ein Memorandum vor. ${ }^{66} \mathrm{Er}$ fand, dass die Ausarbeitung Fricks weitgehend mit den Protokoll-Akten identisch war. Zu kritisieren hatte er nur Folgendes:

1.) Inzeststrafen: a.) Bei den Strafen für Inzest sei beschlossen worden, diese auf ein Drittel herabzusetzen. Im Frickschen Entwurf sei das aber nur für die Geldstrafen geschehen, nicht aber für die Zuchthausstrafen.

b.) In dem ursprünglichen Protokoll heiße es, die Strafe solle auf ein Drittel (von 150 auf 50 Gulden) herabgesetzt werden. Im Frickschen Entwurf stehe nun, sie solle um ein Drittel herabgesetzt werden (von 150 auf 100 Gulden). Da dies der Kirchberger Intention entspreche, sollte es so stehen bleiben.

c.) Bei der Reduktion der 100-Gulden-Strafe sei nicht genau gerechnet worden: Zwei Drittel von 100 seien 66 Gulden und 40 Kreuzer und nicht 66 Gulden. Der 
Unterschied betrifft eine Kleinigkeit, und vielleicht kommt der Fall in Jahrhunderten oder auch gar niemals vor, indessen wird es doch, damit die Bestimmung mit dem angenommenen Grundsaz harmoniere, zu erinnern sein.

2.) Frick habe an einigen Stellen Verwahrungen eingefügt, die besagten, dass die vorgesehenen pragmatischen Lösungen nichts an den jeweiligen Rechtsstandpunkten änderten. Knapp äußerte sich lediglich ironisch über die von Comburg angewandte eigenthümliche Kunst-Sprache, die in der Sache nichts bringe, und dieses Vergnügen könne man den Kontrahenten unbedenklich gönnen.

3.) Knapp brachte dann noch mehrere kleinere Punkte an, die er mit Frick besprechen wollte. Einer erwies sich als belanglos, da er auf einem redaktionellen Versehen beruhte. Wichtiger war die Frage, was geschehen sollte, wenn Kirchberg einen Soldaten nach Mistlau verlege, um das liederliche Bettlersgesindel ausscheu zu machen. In diesem Falle sollten die Verpflegungskosten von den Comburger Untertanen zu bestreiten sein.

4. Comburg hatte selbst angeregt, dass - sollte der Vergleich zustande kommen - dieser auch auf den comburgischen Untertanen in Dörrmenz ausgedehnt würde. Das befürwortete Knapp.

Da Frick ein persönliches Treffen wünschte, schlug Knapp vor, ihn zur Mittagstafel nach Kirchberg einzuladen. Assessor Diezel sei damit einverstanden.

Der Fürst stimmte in einigen Randnotizen zu und wollte Frick gern zum Mittagessen einladen, so dass das Memorandum am 8. Juli 1800 nach Comburg abging. ${ }^{67}$

Bemerkenswert ist, dass in der Akte zum ersten Mal etwas von den damaligen politischen Verhältnissen zu lesen ist. Frick musste die Einladung des Fürsten ausschlagen. Er schrieb am 14. Juli an Knapp, dass nach denen eingetrettenen wüsten Zeitumständen, wo die Feindestruppen sozusagen bis an die Thore unsers Ritterstiftes streifen, die Stadt Hall eine Zeitlang mit 25 Mann besezt gehalten, dermahlen aber sich jedoch nur auf anderhalb Stunden entfernt halten, er außer Stand sei, der Einladung zu folgen. Frick hoffte aber, dass sich die Umstände - also die Anwesenheit französischer Truppen - bald so ändern, dass er den Besuch in Kirchberg nachholen und Vorschläge für den Vertrag machen könne. ${ }^{68}$

Frick meldete sich erst am 10. Januar 1801 wieder bei Knapp. ${ }^{69}$ Er habe eigentlich die Weihnachtsferien zu einem Besuch in Kirchberg nutzen wollen, leider sei aber zur Jahreswende so viel zu erledigen gewesen; das normale Geschäft sei liegen geblieben. So muss es wieder beim Schriftlichen bleiben. Frick nahm Stellung zu Kritikpunkten an seinem Vertragsentwurf, die er im Großen und Ganzen akzeptierte. Dann kam er auf eine Reihe Punkte zu sprechen, die nichts mit dem Vergleich zu tun hatten, aber die Verwaltungen beschäftigen, nämlich

67 StA Ludwigsburg B 375 L Bü 112.

68 AN 69.

69 AN 70. 
wie man das liederliche Bettelgesindel fernhalten könne, und ob man den Gemeindewasen auf die Gemeindebürger verteilen solle, wie bei Teuerungen zu verfahren sei und anderes. Man spürt deutlich den Krieg als Hintergrund. Den Vorschlag, den Vergleich auch auf Dörrmenz und Lendsiedel auszudehnen, befürwortete er, musste dies aber der Ratifikation seiner Herrschaft vorbehalten.

Knapp antwortete am 13. Januar und meinte, dass er mit dem Vergleichs-Prozeß wegen Mistlau vollkommen einverstanden sei, so dass dessen Vollzug nichts mehr im Weg stehe. Danach folgte eine lange Erörterung anderer Fragen, die den Verwaltungen damals auf den Nägeln brannten, aber nichts mit Mistlau zu tun hatten. Weitere schriftliche Erörterungen erschienen ihm nicht nützlich, er hoffte vielmehr, bei einem Treffen seinen Verhandlungspartner bald persönlich zu sehen. ${ }^{70}$

Daraus wurde wieder nichts. Knapp meldete sich erst wieder am 7. September 1801 bei Frick, ${ }^{71}$ mit der Entschuldigung: Durch die Stürme, welche die Reichs-Ursachen alle Augenblicke herbeyführten, wurde man gehindert, andere Geschäfte immer so zeitig in Ordnung zu bringen, als es außer dem zu wünschen gewesen wäre. Dies ist wohl beiderseits der Fall mit den Vergleichen Mistlau, Gaggstatt betr.

In Absicht auf Mistlau ist ohnehin kein Anstand mehr vorhanden, und was Gaggstatt und die sonstigen Gegenstände betrift, worüber man sich bereits wechselseitig genaehert hat, so wird ein gänzlicher Abschlu $\beta$ wohl ebenfalls keine erhebliche Schwierigkeiten mehr finden. Deshalb fragte er an, ob Comburg Interesse hätte, die Sache zu einem gedeihlichen Ende zu bringen.

Eine Antwort auf diesen Brief liegt nicht vor. Die reichspolitischen Ereignisse setzten dem jahrzehntelangen Hin und Her ein Ende. Ein Jahr später, am 9./10. September 1802, marschierten württembergische Truppen auf die Comburg und besetzten sie; am 23. November 1802 nahm Württemberg Comburg formell in Besitz. Damit war Hohenlohe-Kirchberg sein Verhandlungspartner abhanden gekommen - wenn noch etwas geschehen sollte, hätte der Vergleich nun mit Württemberg abgeschlossen werden müssen. Ob dies tatsächlich geschehen ist, ist unklar. ${ }^{72}$

Nur Seckendorff hatte noch nicht genug von solchen Auseinandersetzungen. 1805 beschwerte es sich, dass aus Anlass einer Schlägerei zwischen einem eigenen und einem Kirchberger Untertan in Mistlau Kirchberg die Untersuchung an sich gezogen habe. ${ }^{73}$

$70 \quad$ AN 71.

71 AN 72.

72 In der Kirchberger Akte HZA Ki 1017 A Nr. 56 findet sich die folgende Nota: Pro Nota! Das Anerkenntniß des zwischen Komburg und Kirchberg in Ansehung des Orts Mistlau laut Projects zu fertigenden Vergleichs Recesses p. geschehenen Austrags von Seiten Würtemberg mittelst AntwortSchreibens von der Oberlandesregierung zu Ellwangen d.d. 13. Aug. 1804 liegt den Acten: die vorseyende Landes Purification mit Württemberg betr. bey. Diese Akte ist nicht auffindbar.

73 AN 75 und 76 
1806 erledigte sich dann der Streit endgültig, als Hohenlohe-Kirchberg von Bayern eingenommen (und 1810 im Rahmen eines Gebietstausches an Württemberg weitergegeben) wurde.

\section{Nachbemerkungen}

Es wird deutlich geworden sein, dass sich Comburg mit Kompromissen viel schwerer getan hat als Kirchberg. Möglicherweise ist das auf die Kollegial-Verfassung in Comburg zurückzuführen. Zum einen mussten sich Dekan und Kapitel einigen; zum anderen das Kapitel selbst. Dieses trat nur in großen Abständen zusammen, da die Chorherren meist ihren Wohnsitz nicht auf der Comburg hatten.

Zum zweiten mutet der gesamte Streit in der Zeit von 1788 bis 1802 grotesk an. 1789 brach die Französische Revolution aus, 1793 begannen die Koalitionskriege zwischen Frankreich und einigen deutschen Staaten, 1796 marschierten französische Truppen auch in Südwestdeutschland ein, ab 1800 überfluteten die napoleonischen Heere Europa. Und da stritten sich zwei Miniatur-Staaten 15 Jahre lange darüber, wer von beiden in einem Dorf mit 120 Einwohnern die Streife durchführen durfte und wem welche - allesamt winzigen - Einkünfte und Rechte zustanden - und man kam nicht einmal zu einem Ergebnis. Allein die Kosten für die Arbeitszeit des eingesetzten Personals und für die immer wieder durchgeführten Tagungen dürften die strittigen minimalen potenziellen Einkünfte aus den betroffenen Orten um ein Mehrfaches überstiegen haben. Da triumphierte die Rechthaberei der jeweiligen Herren, aber auch die Eigendynamik einer schreibfreudigen Bürokratie über sämtliche ökonomischen Überlegungen. Wenn es um Hoheitsrechte ging, zählten die damit verbundenen Kosten wenig. Insofern scheint das Ganze ein Paradebeispiel für Bürokratie und Entscheidungsunfähigkeit des Alten Reiches zu sein. Gewiss ist so ein Pauschalurteil nicht ganz falsch. Andererseits zeigt der Fall aber auch, dass im reichsrechtlich gesicherten Rahmen des Reiches sogar kleine und kleinste Herrschaften - ohne von den großen Nachbarterritorien erdrückt zu werden - ohne militärische Gewalt oder andere Willkür in geordneten Bahnen Konflikte auf dem Verhandlungsweg zu lösen versuchten. Der - trotz kontroverser Interessenlage - zivilisierte, manchmal fast freundschaftliche Umgang der Beteiligten miteinander ist letztlich ein positives Beispiel für Konfliktentschärfungsstrategien im Alten Reich. Deshalb ist das Vorstehende mehr als nur eine Aneinanderreihung kleinlichen bürokratischen Umgangs. Es zeigt vielmehr, wie das Alte Reich in seinen letzten Jahrzehnten funktionierte. Und bei aller Langsamkeit: Das Reich funktionierte auf dieser kleinen Ebene immerhin insofern, als Gewalt vermieden wurde. 


\section{Anhänge}

\section{Anhang 1:}

14. Mai 1788: Memorandum von Fürst Christian Friedrich Carl zu Hohenlohe-Kirchberg zu einem tags zuvor eingegangenen Brief des Comburger Dekans Johann Godefried Lothar Franz Freyherr von Greiffenclau zu Vollrats

Gestern habe ich das hier anliegende, unerwartete, an sich ganz höfl. Schreiben von Comburg durch den Postwagen erhalten.

Es gedenkt dieses bloß einiger - in Mistlau - zum theil noch vor meiner Regierung, sich ereigneter unangenehmer Auftritte, und übergehet die Differenzien wegen Gaggstatt (vermutl., weil diese schon am $C[$ ammer $] G[\mathrm{ericht}]^{74}$ eingeklagt und Oberst-Richterlicher Erkändtnis unterworfen worden sind) mit Stillschweigen, - erwähnet die - meiner Regierung wegen jener gemachten - aber den erwünschten Eindruck nicht erwürkten Vorstellungen, auch, nebst dem Wunsch das Nachbarwohlvernehmen fortzupflanzen, der jenseitigen Obliegenheit, wohl hergebrachte Gerechtsame handhaben zu müßen, enthält dann die Bitte, meiner Regierung zu befehlen, wie sie künftig sich benehmen solle und endigt sich mit einem - mir wegen meiner Billig- und Gerechtigkeits-Liebe gemachten Compliment.

Da ich nun dieses Schreiben in höflich und moderatem Ton beantworten - die meinen Gesinnungen wiederfahrende Gerechtigkeit verdanken - und meiner bißher erwiesenen Aufmerksamkeit, dem H. Dechant u. sämtl. H. Capitularen von meiner Hochachtung und gut nachbarl. Gesinnungen bey jeder Gelegenheit, zu überzeugen, Erwähnung thun - zugl. aber auch beedes, mein Bedauern und meine Verwunderung darüber, dass man sich jenseits zu manch thätigen Turbationen meiner Gerechtsame hat verleiten laßen, äußern will: So entstehet hirbey die Frage: ob man den Comburg. Erlaß an mich, als einen solchen Schritt betrachten und aufnehmen dörfe und solle, wodurch man Gelegenheit theils sucht, theils geben will, nicht nur die benannte Irrungen, sondern alle obwaltende auf gütliche Art zu beseitigen $u$. abzuthun?

\section{Anhang 2:}

14. Mai 1788: Stellungnahme des hohenlohischen Assessors Johann Friedrich Widmann zur comburgischen Anfrage wegen Gaggstatt

Das an Eure Hochfürstl. Durchl. von Dechant und Kapitul zu Komburg eingekommene Schreiben ist allerdings eine unvermutete Erscheinung, wobey ich aber eine weniger lautere Absicht, als Hochf.[ürstliche] D.[urchlaucht] selber vermuten, angenohmen. 
Randglosse des Fürsten: ich versire auch in dubio [ich neige im Zweifel dazu], dann doch auch lieber jedem etwas gutes als etwas schlimmes zu[zutrauen].

[...] Wer den Geist der Komburgischen Erweiterungs Systems nur zur Hälfte aus den alten Akten kennt, wer die eigene hartnekige Art weiß, wie sie die sonderbarsten Anmaßungen behaupten und gegen alle noch so gründliche Beweiße zu souteniren suchen, wer einige Wissenschaft von ihren Streitigkeiten mit Hall, Limpurg Württemberg $p$. hat, gegen welche Nachbarn sie bey der sorgfeltigsten Wachsamkeit doch noch im Ganzen immer gewonnen haben, der wird leicht begreifen, dass der Geist des Friedens nicht auf diesem Convent ruhe und dass ihnen mehr darum zu thun sey, unter zweideutigen Behauptungen ihre Zuständigkeiten immer weiter auszudehnen, als durch einen bündigen Receß ihren Absichten eine entscheidende Gränze sezen zu lassen.

Randglosse des Fürsten: als sehr gefährliche Nachbarn so wohl wegen ihrer Grundsäze, als wegen ihrem Vermögen diese durchzusezen, sind sie immer zu betrachten. Man hat also diesseits, wo es an Macht und genugsamem Nachdruck fehlet, umsomehr besonders klug und vorsichtig zu handeln.

Es bleibt ein wahres politisches Phenomen, wenn man die Geschichte dieses Stifts, das ahnhin ein armseliges Benedictiner Convent war, bis zum gegenwärtigen Zeitpunkt verfolgt, wie solches bey seinem anfänglich geringen Umfang, wo es zu erst bald unter Reichsstadt Hällischen, bald unter Hohenlohischen und letztlich unter Limburgischem Schirm gestanden, zu der gegenwärtigen Größe und Stärke angewachsen und sich nunmehr selbst gegen dieienigen furchtbar macht, deren Schuz es ahnhin so angelegen sich gesucht hat. Freyl. hat es einen großen Theil seiner Vergößerung und Stärke der bekannten Connexion mit Würzburg zu verdanken, aber auch so bleibt ihr Betragen ein Meisterstück von schlauer Politik, wenn es mit diesem Stand wegen seiner der Superioritaets Rechte in täglichem Kampf steht und dessen Kräfte gegen Dritte doch immer mit dem Glück zum Erfolg zu bringen weiß, worinnen zugleich ein neuer Grund liegt, warum sie, das Stift, es lieber auf ungewisse Behauptungen, als auf bindige Verträge ankommen laeßt, indem es in extremo doch immer auf Würzburgische Vertrettung und Beystand, wenn er auch nicht zu ausdrückl, wie ahnhin ist, rechnen darf.

Dass also Friedenswünsche bey dem Schreiben zum Grund liegen, zweifle ich sehr. 
Anhang 3:

\section{August 1791: Antwortschreiben des comburgischen Syndikus Weis an den hohenlohischen Rat Cunradi}

[...] In dieser Lage bleibt mir nichts, als das Bedauern übrig, die Mühe bisher umsonst angewandt, und das Maas aller Nachgiebigkeit umsonst erschöpft zu haben, besonders, da allerhand gezogene Sequelae ${ }^{75}$, wie oben gemeldt, das Ritterstift in Verlegenheit sezen.

Das Haus von Seckendorf p:p: sind ganz gewißer Nachricht zu folge mit dem Receßlauf unzufrieden, und die hiesige Unterthanen zu Mistlau senken den Kopf. Ob bey dieser Situation das Verzögern zweckmäßig seye, stelle ich der jenseitigen Klugheit anheim, mit vollkommenster Hochachtung verharrend Euer Wohlgebohrn ergebnster Diener Lt. Weis.

\section{Anhang 4:}

\section{November 1791: Schreiben des comburgischen Syndikus Weis an den hohenlohischen Rat Cunradi}

Dieselbe werden hieraus ersehen, dass die Ritterschaftl. Ganerben [Seckendorff und Hornberg] noch mehrere Alterationen [Änderungen] in der Sache gemacht haben. Euer Hochwolgeboren können daraus auf meine Empfindungen schließen, da sie theils eine Correction für mich waren, besonders wenn ich eine gewiße Art von Sophicismen [Spitzfindigkeiten] betrachte, womit die Monita [Kritikpunkte] begleitet gewesen sind, anderen theils bey dem Pleno Capituli [Kapitelversammlung] die Sensation [das Gefühl] erweckten, welches eben die Ursache meiner Verspätung ist, indeme ich schon entschlossen war keine Hand mehr an die Sache zu legen. Indeß bleibt es immer in der Hauptsache beym alten, und es kann ohnbedenklich ex parte Kirchberg mit beede Hande zugegriffen werden, da es secundaria sind, jedoch soviel erhellet, dass meine Nachgiebigkeit größer, als jene der Ganerben groß wäre.

Uebrigens ist die Sache indivisible [unteilbar] wenigstens in den Haupt Artikeln, so dass wir uns anschließen müßten wir wollten dann, welches nie geschehen wird, die bestrittenen Auslagen pro Litis Consortibus [für die Prozessführung der Konsorten] fahren lassen. Ich sehe einem Empfangsschein entgegen und habe die Ehre, mit vollkommenster Verehrung zu erharren Euer Hochwolgeborn ganz ergebenster Lt. Weis 